\title{
Piperacillin-tazobactam-induced cholestatic jaundice: a report of 2 cases with brief literature review
}

\begin{abstract}
Here we report 2 cases of drug-induced cholestatic jaundice apparently related to piperacillin-tazobactam antibiotic and review briefly the related literature.
\end{abstract}

Keywords: jaundice, drug-induced, cholestasis, piperacillin, Tazobactam
Volume 9 Issue 2 - 2018

\author{
Abdulwahhab Shaheed Abdullah \\ Al-Mustansiriya College of Medicine,Al-Yarmuk Teaching \\ Hospital, Iraq
}

\begin{abstract}
Correspondence: Abdulwahhab Shaheed Abdullah,
Gastroenetrologist, Al-Mustansiriya College of Medicine, AlYarmuk Teaching Hospital, Baghdad Iraq, Email

habeezwed@gmail.com
\end{abstract}

Received: December 23, 2017 | Published: March 16, 2018

\section{Introduction}

Here I report two cases of apparently drug-induced cholestasis caused by the relatively frequently prescribed antibiotic piperacillintazobactam. The first patient is a 52-year-old male who initially presented with right upper quadrant pain, jaundice and dark urine over several days. No fever was reported. Physical examination showed a healthy looking middle-aged man with a tinge of jaundice. Vital signs were normal and only mild right upper abdominal tenderness was evident. Laboratory tests results revealed a normal complete blood count, total serum bilirubin of $3.6 \mathrm{mg} / \mathrm{dl}$, direct serum bilirubin of $2.4 \mathrm{mg} / \mathrm{dl}$, elevated alkaline phosphatase, mildly elevated transaminases, and normal prothrombin time. Abdominal ultrasound showed a moderately-dilated common bile duct (CBD) containing multiple small stones together with innumerable variable sized gallbladder stones. The patient was admitted to the hospital and given prophylactic iv piperacillin-tazobactam $2.25 \mathrm{~g}$. Endoscopic retrograde cholangiopancreatography (ERCP) was then done with sphincterotomy and bile duct stones extraction and plastic biliary stent $12 \mathrm{~cm}$ x $10 \mathrm{Fr}$ was inserted to be removed later after cholecystectomy. The patient was discharged home well on the next day. Two days later the patient attended again with increasing jaundice, itching, together with fever, sweating and rigors. The patient was admitted to the hospital and started on iv fluids and piperacillin -Tazobactam $2.25 \mathrm{~g}$ iv every 6 hours. Investigations showed a neutrophil leukocytosis on blood count, total serum bilirubin of $5.5 \mathrm{mg} / \mathrm{dl}$, direct serum bilirubin of $3.5 \mathrm{mg} / \mathrm{dl}$, and normal prothrombin time. A new ultrasound study reported a mildly dilated CBD with aerobilia with mildly dilated left hepatic duct. Magnetic resonance cholangiopancreatography (MRCP) showed the same result as ultrasound with no features suggestive of sclerosing cholangitis. A test panel for viral and autoimmune liver markers was negative. A second ERCP was done. The stent was obviously functioning but was removed to perform a cleaning balloon sweep which was repeatedly negative with unremarkable occlusion cholangiogram. Bile was clear with no visible pus. A new plastic biliary stent was inserted. Piperacillin-Tazobactam was then stopped and iv Meropenem 1 $\mathrm{g}$ tid was started and fever abated over several days, but jaundice and itching continued to worsen after discharge over several weeks and serum total bilirubin reached 56 $\mathrm{mg} / \mathrm{dl}$ (mainly direct) with very high alkaline phosphatase and nearly normal transaminases. Prothrombin time was normal. Gradually thereafter the jaundice started to decrease until it disappeared over 3 months, the plastic biliary stent was then removed, and laparoscopic cholecystectomy was done, and the patient was well with normal liver function tests and normal biliary tree on ultrasound.

The second patient is a 39-year-old man who was referred for ERCP after several days of right upper abdominal pain and mild jaundice with dark urine. No fever was reported. Physical examination showed a healthy looking adult male with a ting of jaundice and mild right upper quadrant tenderness. Laboratory tests showed a normal total bold count, serum total bilirubin of $2.3 \mathrm{mg} / \mathrm{dl}$ (mainly direct), elevated alkaline phosphatase, and mildly elevated transaminases. Prothrombin time was normal. Abdominal ultrasound showed a mildly dilated CBD with multiple small CBD stones together with multiple gallbladder stones. MRCP (ordered by the referring surgeon) showed the same result as ultrasound. The patient was admitted to the hospital and given prophylactic iv piperacillin-tazobactam $2.25 \mathrm{~g}$. ERCP was then done with sphincterotomy and balloon extraction of multiple small CBD stones. The patient was discharged well the next day. Several days later the patient attended with deep jaundice and itching without pain or fever. Investigations showed a normal total blood count, serum total bilirubin of $14 \mathrm{mg} / \mathrm{dl}$ (mainly direct), very high serum alkaline phosphatase, and near normal transaminases. Prothrombin time was normal. Repeat ultrasound scan showed a completely normal biliary tree and CBD. A new MRCP also showed a completely normal biliary tree and CBD. A test panel for viral and autoimmune liver markers was negative. The patient became anorexic and lethargic and the jaundice and itching continued to worsen reaching a peak total serum bilirubin of $36 \mathrm{mg} / \mathrm{dl}$ (mainly direct) with very high alkaline phosphatase but prothrombin time continued to be normal. Transaminases were nearly normal. Jaundice and other symptoms started to improve gradually until all disappeared over 3 months.

Both patients above received 2-3 doses of iv piperacillin-tazobactam $2.25 \mathrm{~g}$ on the day before the first ERCP procedure as a prophylactic antibiotic. Apart from that, no other drug therapy was given before the development of severe cholestasis. The first patient who reached the highest total serum bilirubin level of $56 \mathrm{mg} / \mathrm{dl}$ also received another 2 or 3 doses of iv piperacillin-Tazobactam $2.25 \mathrm{~g}$ when he was readmitted with features of bacterial cholangitis before he was switched later to iv meropenem. Both patients received corticosteroids (oral 
prednisolone) and ursodeoxycholic acid for several weeks during their course of cholestatic illness without noticeable benefit and were then stopped. Liver biopsy was discussed with both patients, but they refused to offer consent. Both patients were well and asymptomatic with normal biochemical liver tests and normal liver ultrasound on follow up more than 1 year later.

\section{Discussion}

Piperacillin-Tazobactam is a parenteral anti-pseudomonal penicillin that have been used as a prophylactic antibiotic before elective ERCP and is recommended by recent Japanese guidelines for this specific indication. ${ }^{1}$ Although piperacillin-Tazobactam have been implicated in causing bone marrow suppression, ${ }^{2}$ there is only one case report for a possible etiology in drug-induced liver injury. ${ }^{3}$ A recent comprehensive review of drug-induced liver injury puts piperacillin in the category of drugs that "did not fulfill at least a possible relationship" with hepatotoxicity. ${ }^{4}$ The liverTox website also only reports rare instances of idiosyncratic liver injury in persons receiving piperacillin without tazobactam. ${ }^{5}$ Although both patients reported here had ERCP done before developing prolonged cholestasis, they had extensive investigations which confidently excluded ERCPrelated complications or persistent extrahepatic biliary obstruction. The first patient initially developed features of bacterial cholangitis together with cholestasis and it was difficult to decide that no further biliary intervention was necessary, while the clinical picture in the second patient was clearer in favor of drug-induced cholestasis. Even though the results of a liver biopsy, if it was available, were unlikely to be pathognomonic of any specific etiology, liver histopathology might have revealed certain features suggestive of drug-induced liver injury. ${ }^{6}$ Lymphocyte stimulation tests, also reported to be somewhat helpful in suggesting drug induced liver injury, ${ }^{7}$ are not available in our hospital. Although frequently quoted as the gold standard for confirming causality in drug-induced liver injury and other adverse drug reactions, rechallenge with the suspected offending drug, is rarely performed in clinical practice or even in research settings, and is regarded impractical, dangerous, and probably non-ethical behaviour from the clinician side. Instead, it has been suggested that clinical and biochemical improvement of the liver condition upon "dechallenge" from the suspected drug, or "inadvertent rechallenge" with the suspected drug, to be a useful evidence for drug-induced liver injury. ${ }^{8}$

Since traditional demanding causality tools such as the RousselUclaf causality assessment method (RUCAM), ${ }^{9}$ have been shown to underestimate the likelihood of drug-induced liver injury. ${ }^{10,11}$ Therefore, I think there is a need to reconsider the classification of piperacillin-tazobactam as a probable cause of idiosyncratic cholestatic liver injury in the appropriate clinical setting.

\section{Acknowledgements}

I would like to thank Dr. Mohammad Abdullah for his motivation for us in the gastroenterology department to contribute regularly to the weekly clinical meeting were this case report was initially discussed as power point presentation, and also all the hospital staff especially the nursing staff in Al-Yarmuk Teaching Hospital in Baghdad-Iraq.

\section{Conflict of interest}

No relevant issues to be declared.

\section{References}

1. Gomi H1, Solomkin JS, Takada T, et al. TG13 antimicrobial therapy for acute cholangitis and cholecystitis. $J$ Hepatobiliary Pancreat Sci. 2013;20(1):60-70.

2. Scheetz MH, McKoy JM, Parada JP, et al. Systematic Review of Piperacillin-Induced Neutropenia. Drug-Safety. 2007;30:295-306.

3. Quattropani C, Schneider M, Helbling A, et al. Cholangiopathy after short-term administration of piperacillin and imipenem/cilastatin. Liver. 2001;21(3):213-216.

4. Björnsson ES, Hoofnagle JH. Categorization of drugs implicated in causing liver injury: Critical assessment based on published case reports. Hepatology. 2016;63(2):590-603.

5. https://livertox.nlm.nih.gov//PiperacillinandTazobactam.htm

6. Kleiner DE, Chalasani NP, Lee WM, et al. Hepatic histological findings in suspected drug-induced liver injury: Systematic evaluation and clinical associations. Hepatology. 2014;59(2):661-670.

7. Maria VAJ, Victorino RM. Diagnostic value of specific T cell reactivity to drugs in 95 cases of drug induced liver injury. Gut. 1997;41(4):534-540.

8. Garc1'a-Corte's M, Stephens C, Lucena MI, et al. Causality assessment methods in drug induced liver injury: strengths and weaknesses. $J$ Hepatol. 2011;55(3):683-91.

9. Benichou C, Danan G, Flahault A. Causality assessment of adverse reactions to drugs- - II. An original model for validation of drug causality assessment methods: Case reports with positive rechallenge. $J$ Clin Epidem. 1993;46(11):1331-1336.

10. Rockey DC, Seeff LB, Rochon J, et al. Causality assessment in druginduced liver injury using a structured expert opinion process: Comparison to the Roussel-Uclaf causality assessment method. Hepatology. 2010;51(6):2117-2126.

11. Takikawa H, Takamori Y, Kumagi T, et al. Assessment of 287 Japanese cases of drug induced liver injury by the diagnostic scale of the International Consensus Meeting. Hep Research. 2003;27(3):192-195. 